\title{
COVID-19 Vaccine Hesitancy: A Cause of Concern in Rural India
}

\author{
Mounika Pydipalli, Pritam Kumar Roy
}

Sir,

The second wave of Covid 19 after causing disruption across various sectors all over India, is finally showing a downward trend in terms of daily active cases. ${ }^{1}$ Now, it is important on part of the central and the state governments not to lower their guard in terms of number of COVID-19 testing centres, Dedicated Covid Care centres, manufacturing Personal Protective Equipment (PPEs) and most importantly the ongoing Covid Vaccination drive. ${ }^{2}$ As our population is more than 1.3 billion, it has to be understood that it would take a lot of time to inoculate the whole nation. In the present scenario, the goal of the Indian government is to vaccinate 300 million people by the end of August 2021. ${ }^{3}$ As of June 17, 2021, more than 260 million doses of the vaccine has been delivered across India ${ }^{4}$ but the decrease in supply, as well as perceived hesitancy among the general public especially in rural areas to get vaccinated, is a real cause of concern. The main challenge on part of the government lies in mobilizing the people in such areas by enabling them to understand the importance vaccination. Also, lower literacy rates and many cultural, as well as social barriers, hamper the vaccination drive, especially in remote areas. Sometimes some odd cases of Adverse Events Following Immunization (AEFI) too act as a barrier.

One of our author had observed a misinterpreted case of Adverse Events Following Immunization (AEFI) following COVID-19 vaccination, which led to many people showing reluctance to get vaccinated. The case was of a 52 years women residing in a tribal area of Chhattisgarh, India. She got vaccinated in one of the vaccination centres in the district. She was under observation for $30 \mathrm{~min}$. After that, along with her son she went to a ration shop to buy some groceries and while returning, there was sudden onset of mouth deviation and immobility in left limbs. Immediately, she was admitted in hospital and was kept under observation. Case history revealed that the patient had history of hypertension and since 2-3 months she was not under regular anti-hypertensive therapy. Apart from this, there was no history of any other comorbidity and no previous history of such episodes. After 2-3 days, her complications had subsided. So, from the clinical point of view, these events might have resulted due to irregularity in consuming antihypertensive medications and not due to COVID-19 vaccine. But this event created fear among her neighbours in the village and the news spread very fast to the nearby villages which increased the reluctance among the common people. The people were on the belief that before vaccination she was healthy but it's because of the vaccine only she has fallen ill. In one more incident in another district, one of the Accredited Social Health Activist (ASHA) was beaten and defamed by a group of villagers and also accused her that she is asking them to get vaccinated in order to fetch money. Incidents like villagers blocking the roads and prohibiting the entry of healthcare workers has been commonly seen as well. Through these events, we can infer the importance of health education as well as health promotion regarding COVID-19 and at the same time delivering important messages regarding Covid Appropriate Behaviour $(\mathrm{CAB})$ to break the chain of transmission of the virus. ${ }^{5}$ The best way to do this in these tribal and rural areas is by incorporating various stakeholders like the ASHAs, Auxiliary Nurse Midwives (ANMs), Mid-Level Health Providers (MLHPs), Anganwadi Workers (AWW), school teachers and local spiritual as well as religious leaders who will easily be able to build rapport with the people without facing any cultural barriers. At the same, it is very important to strengthen the Health and Wellness Centres (HWCs) and the Anganwadi Centres which will be pivotal in spreading awareness regarding vaccination and $C A B$. In addition to this, another way to increase efficiency is through Inter-sectoral Coordination which can be done by incorporating various other departments to work in a coalition. Active involvement of the district administration is very important for proper planning, implementation, monitoring and supervision of all the activities. Success at the district level will ultimately set an example for the whole State and the end for the entire nation.

\section{REFERENCES}

1. Bhowmick N. Why India's assive COVID-19 surge is breaking tragic records [internet]; 2021. Science. Available from: https://www.nationalgeographic.com/science/article/ how-indias-second-wave-became-the-worst-covid19-surge-in-the-world [cited 19/7/2021].

2. Seethalakshmi S. Exclusive: we cannot lower the guard and succumb to third wave of Covid-19 in India, says Dr Devi Shetty. Khaleej Times; 2021.

3. Bhattacharya A, Shendruk A. India's Covid-19 vaccination program is alarmingly behind schedule. Quartz India. 2021.

4. Ministry of Health and Family Welfare, CoWIN [internet] Available from: https://www.cowin.gov.in/home [cited 19/7/2021].

5. Covid-appropriate behaviour biggest social tool to break the chain of transmission: Harsh Vardhan | India News - Times of India. Times of India [internet] [cited Jun 17 2021]. Available from: https:// timesofindia.indiatimes.com/india/covid-appropriatebehaviour-biggest-social-tool-to-break-the-chain-of-transmission-harsh-vardhan/articleshow/82103760.cms.

Cite this article : Pydipalli M, Roy PK. COVID-19 Vaccine Hesitancy: A Cause of Concern in Rural India. Int J Med Public Health. 2021;11(4):217. 